\title{
Incorporating educational software into large introductory psychology lectures and labs
}

\author{
SARAH RANSDELL \\ New College of the University of South Florida, Sarasota, Florida
}

\begin{abstract}
Psychabilities, a set of 13 computer activities for the introductory psychology course, is introduced. The emphasis of this paper is on using this software, and others of its kind, to coordinate the learning environments of the classroom and the laboratory. The classroom mode in Psychabilities is specifically designed to stimulate discussion during class time while supporting existing lecture-format material. The laboratory mode covers the same content but presents relatively more introductory material to the main learning activity than does the classroom mode. Each module in both modes contains a set of key terms, the main activity, and a set of questions for review. Using educational software more pervasively, that is, in lab as well as in lecture, provides "dual coding" of course content and makes more efficient use of simulations and tutorials that are based on the overall educational needs of introductory psychology students.
\end{abstract}

Large sections of introductory psychology courses are often plagued by an abundance of passive students. Educational software has been developed to increase active learning in the laboratory, especially in methodology courses (e.g., Perone, 1991; Petty \& Rosen, 1990). However, little courseware use or development has focused on improving the relative inactivity of students during large classroom lectures (Ransdell, 1989). This paper describes a package of 13 computer activities designed for classroom and laboratory use to help promote active learning in both environments. The package is called Psychabilities because it combines direct experience with psychological phenomena with the possibilities inherent in interactive educational software. One as yet untapped possibility is to use software to stimulate discussion during class while supporting existing lecture-format material and the coordination of lecture and lab activities.

The first section of the paper distinguishes the features of the two modes of use and includes descriptions of how the classroom mode might be used within class lecture time as compared with how it might be used in the laboratory component of a course. The second section describes the common format of each module in the package and some of its unique features. Finally, availability of the software and its hardware requirements are given in the third section.

\section{Classroom and Laboratory Modes of Use}

Time can seem short during a typical 50-min lecture in general survey classes. Some instructors may be worried about adding anything to that precious time period because it is completely filled by their carefully wrought

The author's address is S. Ransdell, Division of Social Sciences, New College of the University of South Florida, Sarasota, FL 34243-2197 (e-mail:dsdaiam@cfrum). notes. Although instructors might not use the software during every class lecture, if it is used every week or so, they may be able to enhance their students' interest and activity-even if that activity is simply raising a hand to respond in a memory experiment during class. Noncomputerized demonstrations are also effective as a means for increasing active learning, but they do not directly reinforce the activities taking place in the computer-lab section of a course.

Psychabilities provides a way to coordinate lab and lecture activities. The idea behind the computer activity is that it provides "dual coding" of the course material. That is, students read about the content in the text, hear about it in the lecture, and take part in an activity using their knowledge. Working through the activity during class often encourages students to do the module in the lab, and vice versa. I frequently introduce a new topic with such an activity, but it is also effective to provide a brief lecture that introduces key terms before the activity. Key terms are available from a hidden menu at any point in both modes.

Each classroom-mode program lasts about $10 \mathrm{~min}$ and is geared for a large audience (low text, high graphics). The instructor is usually the primary respondent, but he/she may solicit choices from the class and may have students respond with simple key choices if called to the front of the classroom. The corresponding lab-mode program lasts from about $30 \mathrm{~min}$ to $1 \mathrm{~h}$, depending on what the student chooses to do, and contains enough text on screen to let the program stand alone.

In the classroom mode, all students in the class respond by, say, a show of hands. Class data or an individual student's data are compared in bar graphs in the modules that collect experimental data. The main difference between the two is that the classroom mode has less text presented on the screen and collects group rather than individual responses where necessary. Less introductory 
material is necessary since the instructor probably includes this information in regular lecture material. A brief instructor's guide also provides specific classroom ideas for each of the modules.

\section{Features and Format of Each Module}

Each module includes a carefully selected activity in which an entire class or an individual lab student becomes engaged. Activities are in the form of experiment simulations that collect real-time data from the students, experiment simulations that present stored data from prototypical responses, and tutorials that demonstrate or describe related concepts from each content area. Topics were selected because they were particularly central and often difficult for introductory students, not because of the ease with which they could be demonstrated by computer.

Other simulation packages available are less likely to cover the semester-long course as completely as Psychabilities. There is roughly one module for each chapter found in introductory texts and instructor syllabi. Each module has a content name and an activity name. The home menu reveals only the activity name initially since some programs work better without bringing attention to the name of the content, for example, cognitive dissonance (see the Appendix for a complete list of the modules).

Each module takes care to emphasize critical evaluation of ideas presented. For example, in both the module on intelligence testing and the one on personality assessment, conclusions are qualified in terms of the advantages and disadvantages of labeling individuals by means of paper-and-pencil tests. In others, such as the one on neurotransmitters and behavior, it is emphasized that the computer simulation is simplifying a much more complicated process. In the module on Piaget's theory of cognitive development, students assign a cognitive developmental stage to a child and then read field notes that qualify the assessment, in this case suggesting a difference between performance and competence.

Usually done after the activity, but available at any point, are multiple-choice questions of three types: definition, application from the activity, and application from a new context. Since many students in large introductory courses are assessed with this type of question, the software prepares them for thinking about the content and the activity in this way. The questions included with each module may be used during class time, perhaps to review for an exam. After a response, correct or incorrect, an explanatory rejoinder is given.

Keep in mind that several computer activities for introductory psychology students are available, and a thorough analysis of locating, reviewing, and selecting them was done by Hornby and Anderson (1988). They have also provided a more recent review of introductory psychology software available to instructors (Hornby \& Anderson, 1990). A particularly noteworthy one (winner of the 1990 EDUCOM Best Psychology Award) is called PsychSim (Ludwig, 1989). Nearly every current introduc- tory text offers some computer ancillaries. One could use many of them in the way I describe here, although they will not have specifically designed dual modes. The key to successful and efficient use of any educational software is for the student to become engaged in an activity that absorbs him/her. One way to promote this absorption is to make the software-based activities more pervasive.

\section{Hardware and Software Requirements}

Currently, Psychabilities is available only for the IBM PC platform. Specific requirements for the system are minimal. Students working in the laboratory mode will need an IBM PC or compatible computer with at least 512K memory, DOS 2.1 or higher, CGA, EGA, or VGA graphics capabilities, a color or monochrome monitor, and at least one $51 / 4$ - or $31 / 2$-in. disk drive or a hard drive. For classroom presentation, the instructor will also need an overhead projection system or a large screen monitor.

This set of 13 content modules was offered free to Houghton-Mifflin introductory psychology textbook adopters and their students beginning in the fall of 1991 . Interested instructors who do not adopt one of these texts should contact their regional Houghton-Mifflin sales representative to find out about purchasing the software.

\section{REFERENCES}

Hornby, P., ANDerson, M. (1988). Using computers in introductory psychology. Behavior Research Methods, Instruments, \& Computers, 20, 180-183.

HornBy, P., ANDerson, M. (1990). A review of software for introductory psychology instruction. Behavior Research Methods, Instruments, \& Computers, 22, 184-193.

LuDWIG, T. E. (1989). PsychSim [Computer program]. New York: Worth.

Perone, M. (1991). Computer-based methodology laboratories: 1. An undergraduate course in experimental psychology. Behavior Research Methods, Instruments, \& Computers, 23, 121-126.

PetTy, L. C., Rosen, E. F. (1990). Increase in mastery levels using a computer-based tutorial/simulation in experimental psychology. Behavior Research Methods, Instruments, \& Computers, 22, 216-218.

RANSDELL, S. E. (1989). A dynamic duo: LCD overhead displays and laptops as instructional aids in introductory psychology. Collegiase Microcomputer, 7, 147-150.

\section{APPENDIX}

Activity and Content Names of the 13 Psychabilities Modules

1. Kid Stuff-Piaget's Cognitive Developmental Stages

2. Mind Matters-Neurotransmitters and Behavior

3. Detective Work-Signal Detection Theory

4. Total Recall-The Serial Position Effect

5. In Self-Defense-Ego Defense Mechanisms

6. Why Lie?-Festinger's Cognitive Dissonance Experiment

7. The Natural Pain-Killer-The Experiment

8. Target or Subject?-Real World Validity

9. Salary or Hourly?-Schedules of Reinforcement

10. Beer then 21-The 4-Card Selection Task

11. Dilemmas in Testing-Intelligence

12. Typecast-Type A-B Behavior

13. DSMing-Classifying Abnormal Behavior 